\title{
Interaction of the opiate and NMDA systems in a spatial learning task
}

\author{
ELIZABETH BOSTOCK, KATHLEEN E. MCKAY, REGAN FONG, and NAN MIN \\ Queens College, City University of New York, Flushing, New York
}

\begin{abstract}
Two experiments were done to determine whether opiate and NMDA systems interact to influence learning in rats. Experiment 1 examined the effects of coadministration of naloxone with MK-801 on acquisition of a radial maze task. MK-801 produced an acquisition impairment extending beyond drug administration. Naloxone, alone or in combination with MK-801, had no effect. Experiment 2 tested the influence of coadministration of naltrexone with MK-801 on a plus-maze task. MK-801 produced an acquisition impairment extending beyond drug adminis tration. Naltrexone alone had no effect. A three-way interaction (MK- $801 \times$ naltrexone $\times$ days) was found, whereby naltrexone antagonized the effects of MK-801 during the period of drug administration but had no influence on the deficit following this period. This suggests that NMDA receptors are important for acquisition, and the influence of opiates is either time limited or secondary to effects of NMDA receptors on acquisition.
\end{abstract}

Long-term potentiation (LTP) is a long-lasting increase of the synaptic efficacy following afferent tetanic stimulation (Bliss \& Lomo, 1973). This increase in synaptic efficacy is manifested as an increase in the size of the excitatory postsynaptic potential evoked by low-frequency stimulation (Bliss \& Lomo, 1973). LTP occurs in a number of regions throughout the brain, but LTP that occurs in the hippocampus has been proposed as a neurophysiological mechanism involved in associative learning ( $\mathrm{Col}-$ lingridge \& Bliss, 1987; McNaughton \& Morris, 1987). The induction of LTP in the dentate gyrus is dependent on the activation of $\mathrm{N}$-methyl-D-aspartate (NMDA) receptors. The NMDA receptor is an excitatory amino acid receptor subtype, most densely concentrated in the CAI region and dentate gyrus of the hippocampal formation (Monaghan \& Cotman, 1985; Olverman, Jones, \& Watkins, 1984). Indeed, the induction of LTP in the dentate gyrus can be blocked by NMDA antagonists, such as AP5 and MK-801 (Bostock, Croll, \& Sharp, 1990; Morris, 1989). However, in the CA3 mossy fiber system of the hippocampus, LTP has been shown to be critically dependent on the activation of opiate receptors and not on the NMDA receptor (Derrick \& Martinez, 1988; Harris \& Cotman, 1986). Thus, there is evidence for the involvement of both opiate and NMDA systems in the induction of LTP in the hippocampus.

Studies investigating the behavioral effects of NMDA antagonists have found that the administration of AP5 and

This work was supported by PSC-CUNY Grant 6-69214 to E. Bostock and General Research Support NIH RR-07064 to Queens College. All authors are associated with the Neuropsychology Program at CUNY. Correspondence should be addressed to E. Bostock, Psychology Department, Queens College, CUNY, 65-30 Kissena Blvd., Flushing, NY 11367.
MK-801 prior to learning impairs acquisition, whereas administration posttraining has no effect (Benvanga \& Spaulding, 1988; Morris, Anderson, Lynch, \& Baudry, 1986; Morris, Davis, \& Butcher, 1990; Robinson, Crooks, Shinkman, \& Gallagher, 1989; Shapiro \& Caramanos, 1990). Although these findings suggest that NMDA receptor antagonists impair acquisition on a number of tasks, the possibility exists that the effects of these drugs on learning are secondary to perceptual and motor impairments that result following administration of these substances (see Keith \& Rudy, 1990, for a discussion of this issue). A number of studies have revealed that the NMDA antagonists AP5, AP7, and MK-801 interfere with the functioning of sensorimotor pathways (Cahusac, Evans, Hill, Rodriguez, \& Smith, 1984; Mondadori, Weiskrantz, Buerki, Petschke, \& Fagg, 1989) in such a way that behavioral impairments may result. However, studies conducted by Robinson et al. (1989) revealed that MK-801 impaired acquisition at doses that did not impair motor ability. Consistent with these findings, Shapiro and Caramanos (1990) found that systemic injections of MK801 impaired acquisition of a spatial task but not performance of a previously learned spatial task.

The opiate peptides have also been shown to be involved in learning and memory processes. It has been consistently demonstrated that posttraining administration of opiate antagonists enhances performance on a variety of tasks (Gallagher, 1982; Gallagher, Bostock, \& King, 1985; Gallagher, King, \& Young, 1983). The effects of pretraining opiate antagonist administration on acquisition are more variable, such that both enhancement (Decker, IntroiniCollison, \& McGaugh, 1989) and impairment (lzquierdo, 1980) of acquisition have been reported. Thus, the effects of pretraining opiate antagonist administration may be more task specific than the effects of posttraining administration on mnemonic processing. 
Given that both NMDA receptors and opiate receptors appear to be involved in learning and memory processes, as well as LTP, it would be of interest to know whether or not the effects of NMDA antagonists on spatial learning are modulated by the opiate system. In support of this hypothesis, Trujillo and Akil (1991) recently demonstrated an interaction between NMDA and opiate systems in the modulation of analgesia, such that animals treated with MK-801 showed less opiate tolerance. This study is supportive of the suggestion that there is an interaction between the NMDA receptor system and opiate system.

It is known that NMDA antagonists impair spatial learning and opiate antagonists enhance spatial learning. Thus, it might be predicted that coadministration of naloxone with MK-801 or naltrexone with MK-801 may attenuate the effects of MK-801. Alternatively, the opiate dependence of CA3 LTP might predict that coadministration may further impair task acquisition.

\section{EXPERIMENT 1}

On the basis of previous literature, pretrial administration of MK-801 is expected to impair acquisition. To examine whether the effects of MK-801 are attributable to a primary performance deficit versus a direct effect on acquisition, we administered drugs to rats prior to the first four of 20 maze acquisition trials. If the deficit produced by MK-801 continues beyond the time of drug administration, such a finding would be consistent with the interpretation that MK-801 administration impairs learning.

If opiate antagonists produce their effects by modulating the acquisition of spatial information, as is proposed for NMDA antagonists, then administration of naloxone + MK-801 should ameliorate deficits produced by MK-801 administration.

\section{Method}

Subjects. The subjects were 80 experimentally naive Long-Evans hooded male rats (Charles River Laboratories, Wilmington, MA). The rats were housed in the animal facility of the Psychology Department at Queens College (Flushing, Queens, NY). The facility was maintained at $68^{\circ} \mathrm{F}$ on a 12-h light:dark cycle (lights on at $0700 \mathrm{~h}$ ). All testing was carried out between 7:00 and 10:00 a.m. The rats were housed singly, with water available ad lib in the home cage for the duration of the experiment. Two weeks prior to behavioral procedures, food was restricted until the animals achieved $85 \%$ of ad-lib body weight. The rats were maintained at this weight throughout the experiment by being fed a premeasured amount of rat chow following each day's testing.

Apparatus. A radial eight-arm maze was used for testing acquisition. A central octagonal platform $(21.5 \mathrm{~cm}$ across) was raised $26.5 \mathrm{~cm}$ above the floor. Eight arms radiated from this central platform. Each arm was $61 \mathrm{~cm}$ long and $7.75 \mathrm{~cm}$ wide, with Plexiglas edges $3.5 \mathrm{~cm}$ high. Food wells, $0.5 \mathrm{~cm}$ deep, were located $0.5 \mathrm{~cm}$ from the end of each arm. The platform and arms were made of wood and were painted gray. The maze was circularly enclosed by an opaque (navy blue) $185-\mathrm{cm}$-high curtain; an umbrella, a travel bag, a fan, and a cardboard box were made available as extramaze cues.

Drugs. MK-801 (a generous gift from Merck and Co.) was dissolved in saline and administered in doses of $0,0.08$, or $0.10 \mathrm{mg} / \mathrm{kg}$ / $0.5 \mathrm{ml}$ s.c. Naloxone (Sigma Chemicals, St. Louis, MO) was dissolved in saline and administered in doses of $0,1.0,2.0 \mathrm{mg} / \mathrm{kg} / \mathrm{ml} \mathrm{i.p.}$
Behavioral procedures. Two weeks prior to maze acquisition, the animals were handled briefly for 5 days. Immediately after these handling sessions, several reward pellets were given to each animal so that they would habituate to the new food. For 4 days following handling, the animals were placed on a $115 \times 115 \mathrm{~cm}$ open field ( $5 \mathrm{~min}$ per day), where reward pellets were available ad lib. Following the final day of open-field training, maze trials commenced. Each animal was run on the eight-arm maze for 20 trials ( 1 per day). Completion of a trial was contingent upon an animal's entry into each of the eight arms. An error was scored as any visit to an arm that had been previously visited within a trial. Once the animal had entered each of the eight arms, the experimenter removed the rat from the maze and returned it to its home cage. If the animal did not enter each arm once, the trial was said to be complete when the animal had entered a total of 16 arms or after $10 \mathrm{~min}$ had elapsed. The animals were said to have reached criterion when they had entered all eight arms with no more than two errors, on three consecutive trials.

Experimental Design. The experiment was a $3 \times 3 \times 5$ (naloxone $\times$ MK-801 $\times$ trial blocks) factorial, with naloxone and MK801 as between-subject factors and trial blocks as a within-subject factor. Each of $\mathbf{8 0}$ animals was assigned randomly to one of nine groups, as indicated in Table 1 . One group served as the vehicle control group and received s.c. and i.p. injections of saline. The remaining eight groups received varying doses of MK-801 alone, naloxone alone, or the two in combination. Selection of drug dosage was based on pilot work.

MK-801 $(0,0.08$, or $0.10 \mathrm{mg} / \mathrm{kg})$ was administered s.c. $30 \mathrm{~min}$ prior to maze trials; naloxone $(0,1.0$, or $2.0 \mathrm{mg} / \mathrm{kg})$ was administered i.p. 2-3 min prior to maze trials. For the groups that received either drug alone, the appropriate dosage of saline was administered in place of the missing drug. In this way, all animals received both an s.c. and an i.p. injection regardless of group assignment. Injections were given prior to the first 4 of 20 maze trials. No injections were given for the remaining 16 trials.

Prior to each trial, the food wells of all eight arms were baited with two reward pellets (20-mg Bioserve pellets, Frenchtown, NJ). Thirty minutes subsequent to the s.c. injection, and immediately following i.p. injection, each animal was placed on the central platform of the maze and covered with a plastic bowl. The plastic bowl was attached to a nylon string that enabled the examiner to lift the bowl and observe performance while outside the opaque screen. Each trial began once the bowl was raised. The arms entered, as well as the time of each entry, were recorded by the examiner. Selection of an arm was noted when the animal's hindquarters passed the line separating the center platform from the arm.

Data Analysis. For the purpose of data analysis, the data for each animal was collapsed into five blocks of four trials each. For each four-trial block, the average number of errors per trial was calculated for each animal. These data were used for all subsequent statistical analyses. The data were analyzed using a $3 \times 3 \times 5$ (naloxone $\times$ MK-801 $\times$ trial block) ANOVA. Post hoc examination of significant differences on individual trial blocks was done using Student Newman-Keuls tests, with significance set at $p<.05$.

In addition, the number of trials to obtain criterion were calculated for each animal. The data were analyzed using a $3 \times 3$ (naloxone $\times$ MK-801) ANOVA to test for significant effects.

Table 1

The Number of Subjects Randomly Assigned to Each Drug Treatment Condition in Experiment 1

\begin{tabular}{cccc}
\hline & \multicolumn{3}{c}{ Naloxone $(\mathrm{mg} / \mathrm{kg})$} \\
\cline { 2 - 4 } MK-801 $(\mathrm{mg} / \mathrm{kg})$ & 0.0 & 1.0 & 2.0 \\
\hline 0.00 & 8 & 10 & 8 \\
0.08 & 7 & 10 & 9 \\
0.10 & 10 & 10 & 8 \\
\hline
\end{tabular}



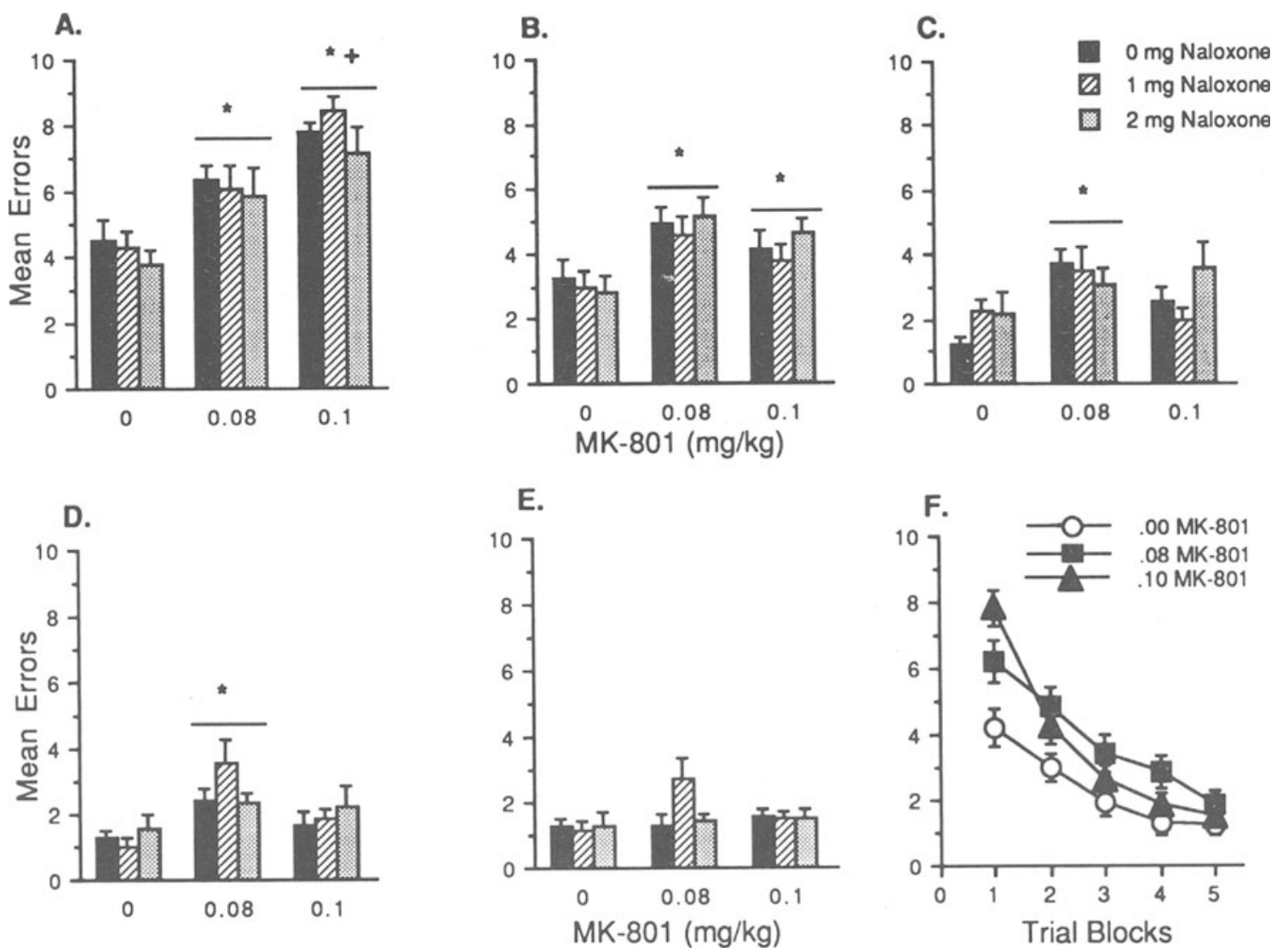

Figure 1. (A-E) Mean errors for rats receiving MK-801, naloxone, vehicle, or naloxone + MK-801 for Trial Blocks 1 (A), 2 (B), 3 (C), 4 (D), and 5 (E). (See Table 1 for numbers of subjects.) (F) Mean errors for rats receiving $0.00,0.08$, or $0.10 \mathrm{mg} / \mathrm{kg}$ MK-801, collapsed across levels of naxolone, for Trial Blocks $1-5(0.00 \mathrm{mg} / \mathrm{kg}, n=26 ; 0.08 \mathrm{mg} / \mathrm{kg}, n=26 ; 0.10 \mathrm{mg} / \mathrm{kg}, n=$ 28). *Groups significantly different from control groups $(p<.05)$. +Groups significantly different from $0.08-\mathrm{mg} / \mathrm{kg}$ groups $(p<.05)$.

\section{Results}

Data from individual trial blocks is shown in Figure 1, which presents mean errors for all groups on Trial Blocks 1-5. Statistical analyses of these data indicate a significant effect of trials $[F(2,284)=142.5, p<.001]$. This can be seen as a reduction of errors across trial blocks. For example, in Figure 1A, the vehicle group is reported to have made an average of 4.5 errors per trial in Trial Block 1, and an average of 3.28 and 1.25 errors per trial in Trial Blocks 2 and 3, respectively.

The data also reveal a significant effect of MK-801 $[F(2,71)=27.8, p<.001]$. This can be seen in Figure 1 when comparing the MK-801 groups with the vehicle groups. Thus, the rats receiving MK-801 made more errors per trial than did the rats that did not receive MK-801.

Also, as can be seen in Figure 1, there was no significant effect of naloxone $[F(2,71)=0.07, p>.9]$, in that animals given naloxone did not differ from their respective controls. Additionally, there was no significant naloxone $\times$ MK-801 interaction $[F(4,71)=0.67, p>.6]$ and no significant naloxone $\times$ MK-801 $\times$ trial block inter- action $[F(4,71)=0.67, p>.6]$. These analyses also demonstrated no significant trial block $\times$ naloxone interaction $[F(8,284)=1.39, p>.2]$ and no significant trial block $\times$ naloxone $\times$ MK-801 interaction $[F(16,284)=$ $1.03, p>.4]$. Analyses did, however, reveal a significant trial block $\times$ MK-801 interaction $[F(8,284)=7.61$, $p<.001]$. The nature of this interaction can best be observed in Figure 1F, which represents mean errors per trial for each level of MK-801, collapsed across levels of naloxone, for each trial block. This figure reveals that animals receiving MK-801 performed worse than did the vehicle controls during the first four of five trial blocks, even though they received MK-801 only during the first trial block.

Post hoc comparisons of the effects of MK-801 administration during individual trial blocks revealed that, on Trial Block 1, animals receiving either 0.08 or $0.10 \mathrm{mg} / \mathrm{kg}$ made significantly more errors than did animals in the vehicle groups. In addition, the animals receiving $0.10 \mathrm{mg} / \mathrm{kg}$ made significantly more errors than did the animals that received 0.08-mg/kg MK-801 (see Figure 1A). In Trial Block 2, animals receiving 0.08- or $0.10-\mathrm{mg} / \mathrm{kg}$ MK-801 


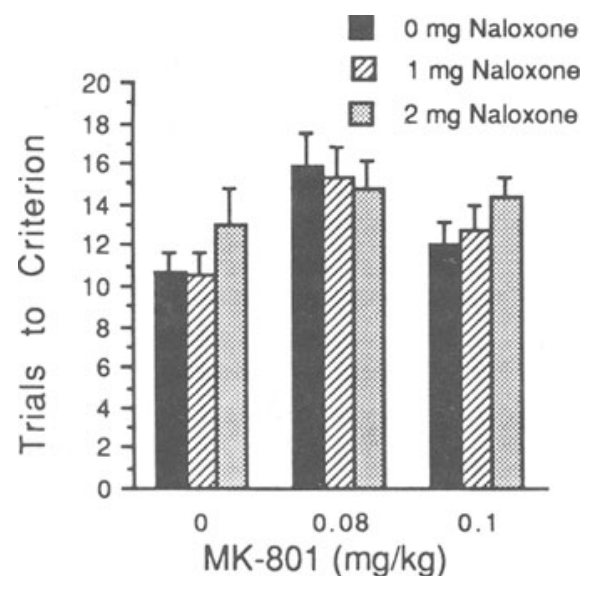

Figure 2. Mean trials to criterion for the indicated groups. For significant differences, see text.

made significantly more errors when compared to the vehicle groups (see Figure 1B). In Trial Blocks 3 and 4, animals receiving $0.08 \mathrm{mg} / \mathrm{kg}$ made significantly more errors than did the vehicle groups (see Figures $1 \mathrm{C}$ and 1D). In Trial Block 5 , there were no significant differences between groups (see Figure 1E).

As with mean errors, analyses of trials to criterion also revealed a significant effect for MK-801 $[F(2,71)=6.45$, $p<.01]$, as shown in Figure 2. However, there was no significant effect of naloxone $[F(2,71)=0.91, p>.4]$ and no significant MK-801 $\times$ naloxone interaction $[F(4,71)$ $=.51, p>.7]$. This effect was such that animals receiving MK-801 required significantly more trials to obtain criterion than did animals that did not receive MK-801 .

\section{Discussion}

As can be seen in Figure 1F, MK-801 impaired performance significantly during the first trial block, when animals received the drug. The motor impairments produced by MK-801 have been shown to be dose dependent, such that $0.08-\mathrm{mg} / \mathrm{kg} \mathrm{MK-801} \mathrm{has} \mathrm{been} \mathrm{reported}$ not to cause motor impairments whereas $0.10-\mathrm{mg} / \mathrm{kg} \mathrm{MK}$ 801 has often been found to cause motor deficits (Robinson et al., 1989). These reports are consistent with our own observations. In this context, it is interesting that $0.08-\mathrm{mg} / \mathrm{kg} \mathrm{MK}-801$, the dose that has been reported not to produce motor deficits, impaired performance not only during the first and second trial blocks but also during the third and fourth trial blocks, well after the time of drug administration. This indicates that animals that receive MK-801 treatment may not have acquired any information during Trial Block 1.

The suggestion that the deficit produced by MK-801 is an acquisition-based deficit is supported by the finding that MK-801 impairs the number of trials required to obtain criterion performance, as seen in Figure 2. Animals in the vehicle group took approximately 10.75 trials to obtain criterion performance, whereas animals in the 0.08 - $\mathrm{mg} / \mathrm{kg} \mathrm{MK-801} \mathrm{groups} \mathrm{took} \mathrm{approximately} 15$ trials to achieve criterion.

Our findings indicate no significant interaction between MK-801 and the opiate antagonist, naloxone, and no significant effect of naloxone alone. This is consistent with the findings of Gallagher et al. (1985), who also found no effect of naloxone on the acquisition of initial training on the radial maze in young and aged rats.

In the absence of a main effect of naloxone, the lack of a significant interaction between MK-801 and naloxone becomes difficult to interpret. One possibility is that the NMDA receptor mechanism and the opiate system are effective at different times. A second possibility is that the opiate system is secondary to the NMDA system and that its activation depends upon successful activation of the NMDA system. Alternatively, it is possible that sufficient time was not allowed prior to testing (2-3 $\mathrm{min})$ for naloxone to enter the system and produce effects.

\section{EXPERIMENT 2}

In Experiment 1, naloxone alone had no effect on original acquisition of the eight-arm radial maze, and no interactions between naloxone and MK-801 were observed. Because the lack of naloxone effects could have been due to pharmacokinetic factors, Experiment 2 examined whether or not the long-acting opiate antagonist, naltrexone, administered $30 \mathrm{~min}$ prior to trials would be capable of modulating MK-801 induced deficits on a spatial learning task.

\section{Method}

Subjects. The subjects were 9 Long-Evans hooded male rats. The animals were obtained, housed, and maintained as described in Experiment 1.

Apparatus. A plus maze was used for testing. A central octagonal platform (21.5 cm across) was raised $26.5 \mathrm{~cm}$ above the floor. A maze arm $(61 \mathrm{~cm}$ long and $7.75 \mathrm{~cm}$ wide, with Plexiglas edges $3.5 \mathrm{~cm}$ high) was extended from four sides of the central platform forming a plus. Food wells, $0.5 \mathrm{~cm}$ deep, were located $0.5 \mathrm{~cm}$ from the end of each arm. The maze was used in four separate rooms for behavioral testing. The room dimensions were: (1) circular $7 \mathrm{ft}$ 2 in. diameter, (2) $11 \mathrm{ft} 3$ in. $\times 7 \mathrm{ft} 4.5$ in., (3) $5 \mathrm{ft} 10.5$ in. $\times$ $10 \mathrm{ft} 4 \mathrm{in}$., and (4) $7 \mathrm{ft} 7 \mathrm{in.} \times 7 \mathrm{ft} 7 \mathrm{in}$. Approximately the same number of extramaze cues were used in each setting.

Drugs. MK-801 was dissolved in saline and administered in doses of 0.0 and $0.08 \mathrm{mg} / \mathrm{kg} / 0.5 \mathrm{ml}$ s.c. Naltrexone was dissolved in saline and administered in doses of 0.0 and $2.0 \mathrm{mg} / \mathrm{kg} / \mathrm{ml} \mathrm{s.c.}$

Behavioral procedures. The animals were trained on a plus maze to locate a goal arm from a randomly chosen start arm. Extramaze cues were available in the environment surrounding the maze. The rats received four trials, at 30-min intervals, per day, 4 days a week for 2 weeks (i.e., 32 trials). The location of the arms was rotated after each trial to minimize the use of intramaze cues. During training, the animals were allowed to roam freely on the maze. They were removed from the maze and returned to their cage if they entered the goal arm, if $5 \mathrm{~min}$ had elapsed, or if $16 \mathrm{arm}$ entries had been made. The arms visited and the time of each entry were recorded by the investigator. Selection of an arm was considered when the animal's hindquarters passed the line separating the center platform from the arm. An error was defined as an entry into a nongoal arm. 
Following initial acquisition, experimental testing commenced. The experimental task was a repeated acquisition task in which spatial learning was tested in four different settings. Thus, each animal was tested under each of the four drug treatment conditions (vehicle, vehicle; MK-801, vehicle; vehicle, naltrexone; MK-801 + naltrexone). The effects of treatment order were controlled for by utilizing a balanced Latin square design. In each setting, the animals were run for two trials per day, at 30 -min intervals, for 8 days. Goal arms for each animal for each setting were randomly assigned, as were the start arms for each trial. MK-801 $(0.0,0.08 \mathrm{mg} /$ $0.5 \mathrm{ml} / \mathrm{kg})$ and naltrexone $(0.0,2.0 \mathrm{mg} / 0.5 \mathrm{ml} / \mathrm{kg})$ were administered s.c. 30 min prior to maze trials for the first 4 days in each setting.

Experimental design. The experiment was a $2 \times 2 \times 2 \times 4$ (MK$801 \times$ naltrexone $\times$ block $\times$ day) within-group factorial design.

Data analysis. For each animal in each setting, the total number of errors per day (over two trials) was calculated. Also, days were broken into two blocks; the first block included the first 4 days, during drug administration, and the second block comprised the last 4 days, during withdrawal (no drugs). These data were used for subsequent statistical analyses. A $2 \times 2 \times 2 \times 4$ (MK-801 $\times$ naltrexone $x$ block $x$ day) repeated measures ANOVA was used to analyze the data.

\section{Results}

The total number of errors per day for all groups is presented in Figure 3. Statistical analyses revealed a significant main effect of MK-801 $[\mathrm{F}(1,8)=15.97, p<.004]$, such that rats made more errors per trial when given MK801 than when given vehicle injections. This effect of MK801 extended beyond the time of drug administration.

A significant effect of block was also found $[F(1,8)=$ 20.78, $p<.0019$ ], whereby rats made more errors in the first block than in the second trial block.

An examination of Figure 3 reveals that there was no significant main effect of naltrexone $[F(1,8)=0.49, p>$ $.504]$. The animals did not differ in the number of errors made per day, whether they were given naltrexone or vehicle. This finding may have been a result of the nature

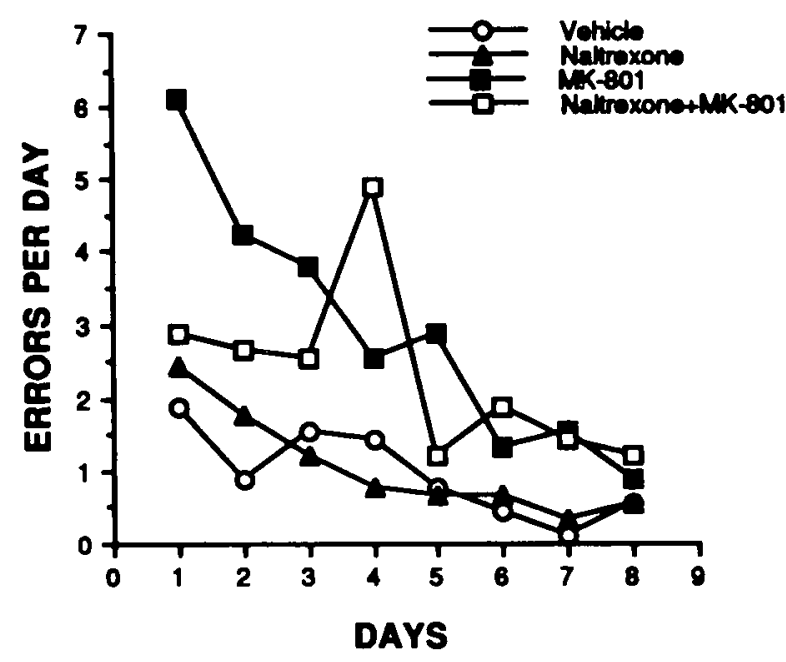

Figure 3. Mean errors per day as a function of days for the indicated treatment conditions. Significant differences are indicated in text. For all treatment conditions, $n=9$. of the task in which it was not sensitive enough to detect significant improvements, thereby producing a floor effect.

Additional analyses revealed no significant two-way interactions. The only significant three-way interaction that was found was MK-801 $\times$ naltrexone $\times$ day $[F(3,24)$ $=4.85, p<.0089]$. The nature of the interaction is such that when given naltrexone $+\mathrm{MK}-801$, the animals made less errors per day than when they were given MK-801 alone, and this difference in performance between the naltrexone + MK-801 and MK-801 treatment conditions disappears as the testing extends past the first 4 days (see Figure 3).

\section{Discussion}

As in Experiment 2, MK-801 had residual effects extending beyond the time of drug administration, such that performance on Days 5-8 in the MK-801 treatment condition is comparable to performance on Days 1-4 in the control condition. As in Experiment 1, this finding suggests that task-relevant information was not acquired while the animals were being treated with MK- 801. Treatment with naltrexone alone had no influence on acquisition relative to controls; however, the performance of controls on this task was very good from Day 1 , so it is possible that the lack of naltrexone effect reflects an inability to detect improvement.

The effects of combined treatment with MK-801 + naltrexone are intriguing. During treatment with MK-801 + naltrexone, the animals made fewer errors than during MK-801 alone treatment, but only for the first 3 days. Beyond this time, the effects of MK-801 + naltrexone and MK -801 alone are essentially the same. This suggests that the ability of naltrexone to exert an influence on this task may be time limited (e.g., opiate systems may only be active during the first 3 days in an environment). Alternatively, the influence of the opiate system on this task may be dependent on a functioning NMDA system. Finally, it is possible that naltrexone is influencing performance rather than mnemonic factors in this experiment. Further research will be required to distinguish between these alternatives.

\section{GENERAL DISCUSSION}

In agreement with Shapiro and Caramanos (1990), who found residual effects during withdrawal after chronic administration of MK-801, the results of Experiments 1 and 2 reveal that MK-801 produced impairment in acquisition during the time of withdrawal in addition to the period of drug administration. These findings argue against the effects of MK-801 being simply a performance-based deficit, as advanced by Keith and Rudy (1990). Rather, these findings are consistent with the interpretation that NMDA antagonists impair acquisition. This suggests that NMDA receptors, at least in some brain sites, may be part of a neural mechanism mediating information acquisition.

The animals that received MK-801 made perseverativetype errors (i.e., going back and forth between two arms) 
while under the influence of the drug. There was no evidence of stereotypic behavior in the animals during withdrawal week, ruling out the possibility that the results obtained were due to poor habits or strategies developed by the rats that interfered with later acquisition. Interestingly, it was observed that the rats that received naltrexone + MK-801 made fewer perseverative-type errors.

As mentioned previously, the lack of naloxone effect in Experiment 1 might have been due to pharmacokinetic factors, whereas the lack of naltrexone effect in Experiment 2 might have been due to a floor effect. However, it is possible that administering opiate antagonists prior to acquisition trials has no influence on acquisition of the tasks utilized. The effects of pretraining opiate antagonist administration are variable (Decker et al., 1989; Izquierdo, 1980), consistent with this notion.

\section{REFERENCES}

Benvanga, M. J., Spaulding, T. C. (1988). Amnesic effect of the novel anticonvulsant, MK-801. Pharmacology, Biochemistry \& Behavior, 30, 205-207.

Buiss, T. V. P., \& Lomo, T. (1973). Long-lasting potentiation of synaptic transmission in the dentate area of the anaesthetized rabbit following stimulation of the perforant path. Joumal of Physiology (London), 232, 331-356.

Bostock, E., Croll, S. D., Sharp, P. E. (1990). Effects of the NMDA receptor antagonist, MK-801, on LTP in freely behaving rats. Society for Neuroscience Abstracts, 16, 263.

Cahusac, P. M. B., Evans, R. H., Hill, R. G., Rodriguez, R. E., a Smith, D. A. S. (1984). The behavioral effects of an N-methylD-aspartate receptor antagonist following application to the lumbar spinal cord of conscious rats. Neuropharmacology, 23, 719-724.

Collingridge, G. L., Buss, T. V. P. (1987). NMDA receptors: Their role in long-term potentiation. Trends in Neuroscience, 10, 288-293.

Decker, M. W., Intront-Collison, I. B., \& MCGaugh, J. L. (1989). Effects of naloxone on Morris water maze learning in the rat: Enhanced acquisition with pretraining but not posttraining administration. Psychobiology, 17, 270-275.

Derrick, B. E., \& MARTinez, J. L. (1988). Naloxone blocks mossy fibre but not commissural or heterosynaptic LTP in the Hippocampal CA3 region in vivo. Society for Neuroscience Abstracts, 14, 565.

GALLAGHER, M. (1982). Naloxone enhancement of memory processes: Effects of other opiate antagonists. Behavioral \& Neural Biology, 35 , 375-382.

Gallagher, M., Bostock, E., King, R. (1985). Effects of opiate antagonists on spatial memory in young and aged rats. Behavioral \& Neural Biology, 44, 374-385.

Gallagher, M., King, R. A., Young, N. B. (1983). Opiate antagonists improve spatial memory. Science, 221, 975-976.

HARRIS, E. W., \& Cotman, C. W. (1986). Long-term potentiation of guinea pig mossy fiber responses is not blocked by N-Methyl-DAspartate antagonists. Neuroscience Letters, 70, 132-137.

IzQUIERDO, I. (1980). Effect of B-endorphin and naloxone on acquisition, memory and retrieval of shuttle avoidance and habituation learning in rats. Psychopharmacology, 69, 111-115.

KeITH, J. R., RUDY, J. W. (1990). Why NMDA-receptor-dependent long-term potentiation may not be a mechanism of learning and memory: Reappraisal of the NMDA-receptor blockade strategy. Psychobiology, 18, 251-257.

MCNaughton, B. L., \& Morris, R. G. M. (1987). Hippocampal synaptic enhancement and information storage within a distributed memory system. Trends in Neuroscience, 10, 408-415.

Monaghan, D. T., Cotman, C. W. (1985). Distribution of NMDAsensitive L-3H-glutamate binding sites in rat brain as determined by quantitative autoradiography. Joumal of Neuroscience, 5, 2909-2919. Mondadori, C., Weiskrantz, L., Buerka, H., Petschke, F., FagG, G. E. (1989). NMDA receptor antagonists can enhance or impair learning performance in animals. Experimental Brain Research, 75, 449-456.

Morris, R. G. M. (1989). Synaptic plasticity and learning: Selective impairment of learning in rats and blockade of LTP in vivo by the NMDA receptor antagonist AP5. Journal of Neuroscience, 9. 3040-3057.

Morris, R. G. M., ANDerson, E., LynCh, A. E., \& BAUdry, M. (1986). Selective impairment of learning and blockade of long-term potentiation by an N-Methyl D-Aspartate receptor antagonist, AP5. Nature, 319, 774-776.

Morris, R. G. M., DAvis, S., BuTcher, S. P. (1990). Hippocampal synaptic plasticity and NMDA receptors: $A$ role in information storage? Philosophical Transactions of the Royal Society of London, 329, 187-201.

Olverman, H. J., Jones, A. W., \& WatKINS, J. C. (1984). L-Glutamate has higher affinity than other amino acids for [3H]-D-AP5 binding sites in rat brain membranes. Nature, 307, 462-465.

Robinson, G. S., JR., Crooks, G. B., JR., Shinkman, P. G., GalLAGHER, M. (1989). Behavioral effects of MK-801 mimic deficits associated with hippocampal damage. Psychobiology, 17, 156-164.

Shapiro, M. L., \& Caramanos, Z. (1990). NMDA antagonist MK801 impairs acquisition but not performance of spatial working and reference memory. Psychobiology, 18, 231-243.

TrujILLo, K. A., A AKIL, H. (1991). Inhibition of morphine tolerance and dependence by the NMDA receptor antagonist MK-801. Science, 251, 85-87.

(Manuscript received February 14, 1991; revision accepted for publication October 17, 1991.) 